\title{
Transplantation therapy for Parkinson disease: the good, the bad and the enigmatic
}

\author{
Oksana Suchowersky
}

Currently, Parkinson disease (PD) affects around 3 per 1,000 individuals worldwide, with an estimated 1 million cases in North America alone. Motor symptoms in PD initially respond well to dopaminergic replacement medications, but the progressive nature of the disorder results in loss of effectiveness of these agents, along with gait dysfunction and complications such as dyskinesias, within 10 years of disease onset.

The search for novel symptomatic and neuroprotective therapies for PD has been an active area of research over the past two decades. One approach has been to use transplantation therapy to attempt to replace the deteriorating cell function, restore dopaminergic stimulation in the basal ganglia, and perhaps even reverse disease progression. Beginning some 20 years ago, transplantation of fetal ventral mesencephalic cells into the putamen was performed at several centers on small numbers of patients in open-label studies, with reportedly good results. These findings generated considerable excitement worldwide, but further studies on larger cohorts of patients in controlled blinded trials failed to confirm the initial reports (Freed CR et al. [2001] N Engl $J$ Med 344: 710-719). PET and pathological analyses revealed adequate survival of grafted neurons, but clinical benefit was seen in only a small subset of patients, and untreatable complications such as 'runaway dyskinesias' became apparent.

As was recently reported in Nature Medicine, clinical and autopsy results are now available on patients up to 16 years after transplantation. Mendez et al. showed survival of grafts without PD pathology for 14 years in five patients (Mendez l et al. [2008] Nat Med 14: 507-509). Another patient described by Kordower et al. had a good clinical improvement lasting for over 5 years, before gradual worsening of motor function and development of gait and balance problems (Kordower $\mathrm{JH}$ et al. [2008] Nat Med 14: 504-506). Autopsy
... not only is

the pathology of PD

progressive,

but it can

also spread

to younger

grafted

neurons

O Suchowersky is an Advisory Board member of Nature Clinical Practice Neurology.

Competing interests

The author declared no competing interests.

www.nature.com/clinicalpractice doi:10.1038/ncpneuro0898 results from this patient 14 years after transplantation showed robust graft survival. However, the grafted neurons had pathological changes typical of PD, including Lewy bodies that stained for $\alpha$-synuclein and ubiquitin. In addition, the grafts were infiltrated by large numbers of activated microglia. Li et al. reported similar graft pathology in three patients up to 16 years after transplantation (Li JY et al. [2008] Nat Med 14: 501-503). These results indicate that not only is the pathology of PD progressive, but it can also spread to younger grafted neurons.

Will transplantation therapy ever be a viable, widespread treatment option for PD? Several issues need to be considered. First, the basal ganglia are a highly integrated and complex set of networks that form gradually over many months during gestation. It seems naive to expect foreign cells inserted into a partially deteriorating system to be able to make the appropriate connections and function in a physiological manner. Second, PD is not just a disorder of the dopaminergic system. The involvement of other neurotransmitter systems results in a variety of nonmotor symptoms, including autonomic dysfunction, mood disorders, fatigue, pain, sleep disorders and, eventually, cognitive deterioration. Dopaminergic cell replacement, even if successful, would treat only the motor symptoms. Last, a number of technical questions still remain unanswered. For example, which type of stem cell-fetal or adult-would be most effective? How many cells should be transplanted and into which location? Should immunosuppression be used?

The recently reported autopsy results raise new concerns regarding the viability of transplantation therapy. Even if clinical motor benefit can be achieved, it could be relatively transient, with the grafts eventually deteriorating owing to the development of PD pathology. The search for improved medical therapies, along with neuroprotective and disease-modifying strategies, must, therefore, continue. 